\title{
Apoptotic and Antiproliferative Effect of Gingiva Mesenchymal Stem Cells on Acute Leukemia T Cells
}

\author{
Deniz Genç,2 $\mathbb{D}$, Merve Sezer Kürkçü² $\left(\mathbb{D}\right.$, Serhat Sezgin $^{3}$ (D) \\ 'Muğla Sıtkı Koçman University, Faculty of Health Sciences, Muğla, Turkey \\ ${ }^{2}$ Muğla Sıtkı Koc̣man University, Research Laboratories Center, Muğla, Turkey \\ ${ }^{3}$ Muğla Sıtkı Koçman University, Faculty of Dentistry Muğla, Turkey
}

ORCID iDs of the authors: D.G. 0000-0003-035I-2805; M.S.K. 0000-0003-0947-29I2; S.S. 0000-000I-7899-8I7I.

Cite this article as: Genç D, Kürkçü MS, Sezgin S. Apoptotic and Antiproliferative Effect of Gingiva Mesenchymal Stem Cells on Acute Leukemia T Cells. Cyprus J Med Sci. 2021; 6(4): 303-310.

\section{BACKGROUND/AIMS}

The aim of this study was to investigate the antiproliferative and apoptotic effect of gingiva-derived mesenchymal stem cells (GMSCs) on the Jurkat cells as t-cell acute lymphoblastic leukemia cell line.

\section{MATERIAL and METHODS}

The Jurkat cells were cocultured with GMSCs or alone at $37^{\circ} \mathrm{C} 5 \% \mathrm{CO}_{2}$ humidified atmosphere with different culture periods and concentrations. The Jurkat cells were subjected to flow cytometry analysis for proliferation, apoptosis, and necrosis by staining the cells with Annexin $\vee$ and 7AAD antibodies. Intracellular IL-2 secretion in the Jurkat cells was analyzed to determine the proliferative cytokine secretion. CD4+CD25+FoxP3+ cells were analyzed to determine the regulatory T cell population. TNFRI and TNFR2 expressions were analyzed for cell death signaling pathways.

\section{RESULTS}

GMSCs significantly reduced the proliferative response of the Jurkat cells in 48 hours of culture period in I:I, I:2, and l:5 (GMSC:Jurkat) ratios. The minimum inhibitory effect on the proliferative response was found to be in 1:5 ratios. GMSCs significantly increased the rate of early apoptosis and necrosis of Jurkat cells in 1:5 (GMSC:Jurkat) ratios. Intracellular IL-2 secretion of the Jurkat cells significantly reduced with GMSCs $(P<.05)$. GMSCs tended to increase CD4+CD25+FoxP3+Tcell population in the Jurkat cells in 24 and 48 hours of culture periods, but no significant difference was observed $(P>.05)$. TNFR2 expression on the Jurkat cells significantly increased within the culture periods when cultured with GMSCs.

\section{CONCLUSION}

This study demonstrated that GMSCs can response to acute leukemia T cells and can modulate the proliferative response by increasing the apoptosis and necrosis and TNFR2 expression and by decreasing IL-2 secretion. Further in vitro or in vivo studies can be performed to investigate the molecular mechanisms or suppressive effects of GMSCs on acute leukemia T cell cells.

Keywords: Gingiva mesenchymal stem cells, T-cell acute lymphoblastic leukemia, apoptosis

\section{INTRODUCTION}

T-cell acute lymphoblastic leukemia, which are aggressive proliferations of transformed T-cell progenitors, account for $10-15 \%$ of T-cell acute lymphoblastic leukemia cases in children and $25 \%$ of adult T-cell acute lymphoblastic leukemia cases.' In addition to current T-cell acute lymphoblastic leukemia treatment, it is important to develop a new strategy to support the apoptosis of lymphoma cells. Most apoptosis inducers currently used in the treatments for T-cell acute lym-

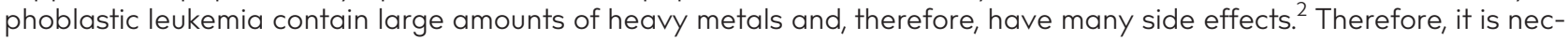
essary to develop new cell-based therapies with lower toxicity.

Gingiva tissue-derived mesenchymal stem cells (GMSCs) are easily accessible multipotent stromal cells originated from oral cavity, which have both anti-inflammatory and anticancer effects as well as its regenerative effects on tissue damages. ${ }^{3,4}$ Although the effects of mesenchymal stem cells (MSCs) on tumor prognosis are uncertain, it has been demonstrated by previous studies that their inhibitory effects on tumor growth processes have been observed, and they have 
been shown to be targeted anticancer agents that can inhibit tumor growth by blocking various tumor processes by regulating the growth of many tumor cells through paracrine mechanisms. $^{5}$

The immunosuppressive properties of MSCs play an important role in preventing cancer progression. MSCs derived from various sources such as bone marrow, adipose tissue, or umbilical cord have been shown to be able to regulate the immune response by affecting the activation, maturation, proliferation, differentiation, and effector function of immune system cells. ${ }^{6}$

Although MSCs have the same morphological features, they develop different immune responses depending on the tissue from which they originate and the inflammatory niche. To date, most studies on the modulatory effect of MSCs on acute lymphoblastic leukemia have mostly been performed with bone marrow MSCs.7 However, the diverse immunosuppressive mechanisms of MSCs isolated from different tissues, uncovering new sources of MSCs that may have higher immunosuppressive capacity will contribute to the expansion of cellular therapy options. Therefore, we investigated the antiproliferative and apoptotic effects of gingiva MSCs on the Jurkat cells as $\mathrm{T}$-cell acute lymphoblastic leukemia cell line by evaluating the antiproliferative and apoptotic responses, FoxP3 expressing $\mathrm{T}$ regulatory cell frequency, and IL-2 secretion by culturing acute $T$ cell lymphoma cells in vitro for the first time.

\section{MATERIAL and METHODS Cell Lines}

The Jurkat cell line (ATCC, Clone E6-I) was used as acute T cell leukemia cell line and obtained from the Muğla Sıtkı Koçman University Research Laboratories Center culture isolates. GMSCs from five healthy donors in the third passage were obtained from culture isolates of the same center. The ethical approval for GMSC isolation was previously obtained from the Muğla Sıtkı Koçman University Clinical Research Ethics Committee (I0/VII 0I.I0.2020).

\section{The Analysis of GMSCs for the Cell Surface Markers}

GMSCs from five donors in the third passage were analyzed for the positive (CD29, CD73, and CDI05) and negative (CD3, CD28, and HLA-DR) cell surface markers for MSCs. In brief, frozen cells were thawed at $37^{\circ} \mathrm{C}$, washed with phosphate buffered saline (PBS) (Sigma-Aldrich, Germany) twice, and centrifuged at 1,500 rpm for 5 minutes. The remaining cell pellet was stained with anti-CD29 (APC), anti-CD90 (PerCp), anti-CDI05 (FITC), anti-HLA-DR (APC), anti-CD3 (PerCp), and anti-CD28 $(P E)$ and incubated at $+4^{\circ} \mathrm{C}$ for 30 minutes. All antibodies were purchased from BD Biosciences, USA. Cells were analyzed via

\section{Main Points}

- Gingiva mesenchymal stem cells (GMSCs) downregulate the proliferative response of T-cell acute lymphoblastic leukemia cells by decreasing IL-2 production.

- GMSCs have an apoptotic effect on T-cell acute lymphoblastic leukemia cell line.

- The activation and proliferation of T-cell acute lymphoblastic leukemia cells can be reduced with GMSCs depending on the cell ratio. flow cytometry for the mean fluorescent index \% (MFI\%) on the Accuri C6 Plus software (BD Biosciences, USA).

\section{Culture Conditions}

GMSCs were separately seeded in 24-well plates with the amount of $5 \times 10^{4}$ cells per well in Dulbecco's modified Eagle's medium (Pan Biotech, Germany) supplemented with 10\% fetal bovine serum (FBS) (Pan Biotech, Germany) and 1\% pencillin/ streptomycin (I00 IU mL $\mathrm{mL}^{-1}, 100 \mu \mathrm{g} \mathrm{mL}^{-1}$ ) (Thermofisher, USA) 48 hours before the coculture. The Jurkat cells were cultured alone or with GMSCs with the ratio of $1: 1,1: 2,1: 5$, and 1:10 (GMSCs:Jurkat cells) suspended in RPMl 1640 medium (Pan Biotech, Germany) supplemented with 10\%FBS (Pan Biotech, Germany) and $1 \%$ pencillin/streptomycin ( $100 \cup \mathrm{mL}^{-1}, 100 \mu \mathrm{g}$ $\mathrm{mL}^{-1}$ ) (Thermofisher, USA) in 24 -well plates at $37^{\circ} \mathrm{C}$ and $5 \% \mathrm{CO}_{2}$ incubator for time periods of $6,12,24$, and 48 hours. $T$ cell stimulation was done with anti-CD3 and anti-CD28 (Thermofisher, USA) 10 and $2 \mu \mathrm{g} \mathrm{mL}^{-1}$, respectively. At the end of each culture periods, the Jurkat cells were collected and analyzed for apoptosis, necrosis, proliferation rate, IL-2 secreting CD3+ cells, FoxP3 expressing CD4+CD25+ T regulatory cells, and tumor necrosis factor receptor I (TNFRI) and tumor necrosis factor receptor 2 (TNFR2) expressions via flow cytometry, as described in the analysis sections. The minimum antiproliferative response was determined by observing the significant decrease in the proliferation ratio in the cocultured cells compared to the Jurkat cell line cultures alone.

\section{Coculture of the Jurkat Cells with GMSCs}

After determining the minimum concentration of GMSCs:Jurkat cells, we cultured the Jurkat cells in the presence and absence of GMSCs or GMSCs with healthy mononuclear cells suspended in RPMI 1640 medium (Pan Biotech, Germany) supplemented with FBS (Pan Biotech, Germany) and 1\%pencillin/ streptomycin (Thermofisher, USA) with the specific T lymphocyte stimulation with anti-CD3 and anti-CD28 (Thermofisher, USA) 10 and $2 \mu \mathrm{g} \mathrm{mL}^{-1}$, respectively, in 24 -well plates at $37^{\circ} \mathrm{C}$ and $5 \% \mathrm{CO}_{2}$ incubator for time periods of $6,12,24$, and 48 hours.

\section{Analysis of Apoptosis of the Jurkat Cells}

To analyze the apoptosis or necrosis of the Jurkat cells, the collected cell suspension was stained using Annexin $V(P E)$ and 7AAD (BD Biosciences, USA) in the room temperature in the dark for 15 minutes. Cells were analyzed via flow cytometry and data recorded as MFI\%. The Jurkat cells were gated from total cell population and gated for $\mathrm{CD}^{+}$cells. Four quadrants were analyzed as follows: lower left quadrant for cell survival, lower right quadrant for early apoptosis, upper right quadrant for late apoptosis, and upper left quadrant for necrosis.

\section{Analysis of Proliferation of the Jurkat Cells}

The Jurkat cells were labeled using Carboxyfluorescein succinimidyl ester (CFSE) labeling kit (Thermofisher, USA) at the beginning of the culture period. Briefly, cells were washed with PBS and centrifuged at 1,500 rpm for 5 minutes. Remaining cell pellet was resuspended in I $\mathrm{mL}$ of PBS, and $5 \mu \mathrm{M}$ of CFSE solution was added in the cell suspension and incubated at $4^{\circ} \mathrm{C}$ for 20 minutes in the dark. Cells were then washed twice with RPMI medium supplemented with 10\%FBS. Thereafter, culture period cells were analyzed via flow cytometry in the FITC channel. The proliferation of the Jurkat cells was detected by gating $\mathrm{CD}^{+}$cell population and analyzed for CFSE signaling 

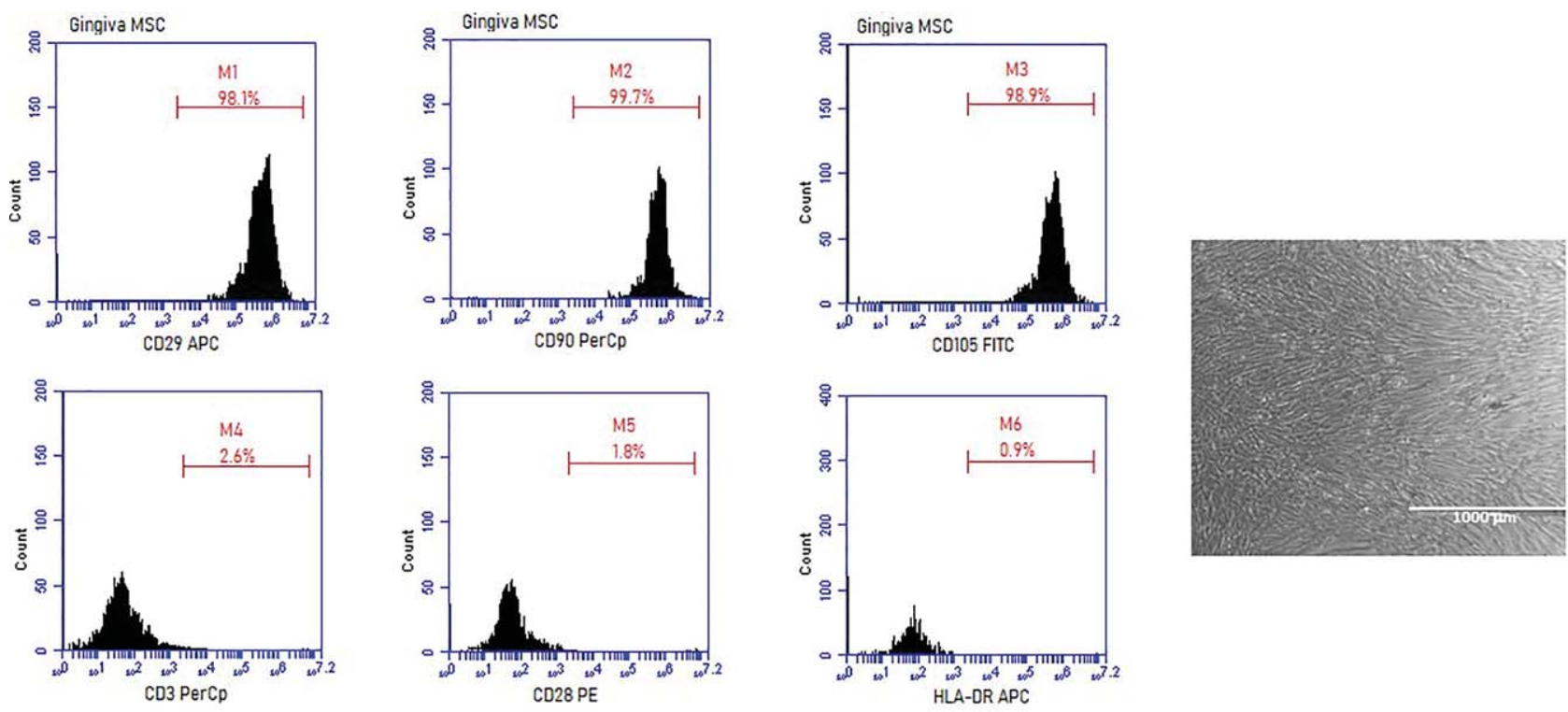

Figure I. Characterization of GMSCs. Flow cytometry analysis for the positive cell surface expressions (CD29, CD90, and CDI05) and negative cell surface expressions (CD3, CD28, and HLA-DR) for mesenchymal stem cells. GMSCs expressed positive cell surface markers over $95 \%$ and lack the expressions of negative markers. GMSCs showed fibroblast-like colonies in the third passage.

via flow cytometry (BD Biosciences, USA). Histogram analysis was performed for proliferation ratio.

\section{Analysis of CD4+CD25+FoxP3+ T Cells}

$\mathrm{T}$ regulatory cell population is a suppressive cell type in immune responses. Therefore, we analyzed CD4+CD25+FoxP3+ $T$ regulatory cells after culture periods. The Jurkat cells were washed twice with PBS, cell surface staining was performed using anti-CD4 (FITC) and anti-CD25 (APC) at $4^{\circ} \mathrm{C}$ for 30 minutes, and intracellular staining was performed using anti-FoxP3 (PE) at $4^{\circ} \mathrm{C}$ for 20 minutes. Cells were analyzed via flow cytometry, and CD25 cells were gated to analyze CD4+FoxP3+ cells. The data were recorded as MFI\% in the dot plot analysis.

\section{Intracellular IL-2 Analysis}

The Jurkat cells were analyzed for intracellular cytokine secretion for IL-2, which induces $T$ cell proliferation. The cells were first stained using anti-CD3 (PerCp) for the cell surface marker of $\mathrm{T}$ lymphocytes and incubated at $4^{\circ} \mathrm{C}$ for 30 minutes in the dark. After washing the cells using PBS, permeabilization buffer was added and incubated for 20 minutes at $4^{\circ} \mathrm{C}$. The permeabilized cells were then stained with anti-IL-2 (APC) (BD Biosciences, USA) antibody for 30 minutes at $4^{\circ} \mathrm{C}$ and analyzed for intracellular IL-2 secreting $T$ lymphocytes. All antibodies were purchased from BD Biosciences, USA.

\section{Analysis for TNFRI and TNFR2 Expression}

TNFRI and TNFR2 signaling pathways are key regulatory factors that generate apoptotic cell death signals in many of the cells. We analyzed TNFRI and TNFR2 expressions on the Jurkat cells at the end of the culture periods. Cells were stained using anti-CDI20 $\alpha$ (PE) for TNFRI or anti-CDI20b (PE) for TNFR2 expressions. Histogram analysis was performed for the expression of TNFRI or TNFR2 for the Jurkat cells via flow cytometry. Histogram analysis was performed for $\mathrm{CD}^{+}$cell population.

\section{Statistical Analysis}

Differences between groups were analyzed using the SPSS program and the Graphpad Prism program version 8.0 (Graphpad Software, Inc., CA, USA). Data were given as mean \pm standard deviation (SD) (minimum-maximum) values in each group. Comparison of the data of two groups was done by one-way ANOVA test. $P<.05$ values were considered significant.

\section{RESULTS \\ GMSCs Showed Positive Cell Surface Markers Expression for MSCs and Formed Fibroblast-Like Colonies in the Culture}

The third passage GMSCs were analyzed for positive and negative cell surface markers expressions to confirm these cells as MSCs. The cells were expressed as CD29, CD73, and CDI05 over $95 \%$ and lack the expressions of negative markers. The cells showed fibroblast-like colonies in the culture flasks (Figure I).

\section{The Proliferation of the Jurkat Cells Reduced with GMSCs}

The proliferation analysis was performed to evaluate the suppressive effect of GMSCs on the Jurkat cells. Five separate cultures were performed for each of GMSCs with the Jurkat cells. CFSE-labeled Jurkat cells were cultured in the presence and absence of GMSCs with the ratio of 1:1, 1:2, 1:5, and 1:10 (GMSCs:Jurkat cells) or Jurkat cells alone, and analysis was done for 6, 12, 24, and 48 hours of the culture periods. The proliferative response of the Jurkat cells significantly increased in 24 hours $(24.6 \pm 5.7)$ and 48 hours $(43.1 \pm 4.8$ ) culture periods compared to 6 and 12 hours of the culture periods (6 hours: $6.4 \pm 2.1$, 12 hours: $10.8 \pm 2.9$ ) $(P<.001$ ). GMSCs significantly inhibited the proliferation ratio of the Jurkat cells in $1: 1,1: 2$, and 1:5 cocultures in 48 hours of the incubation period 


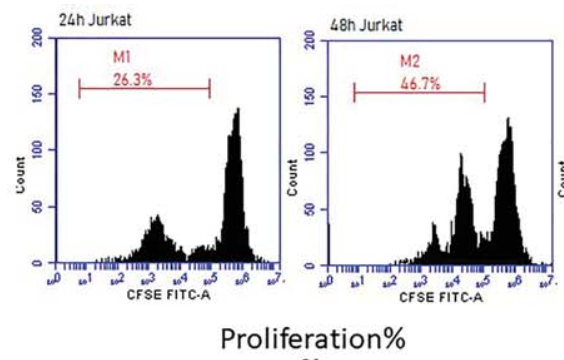

$6 h$

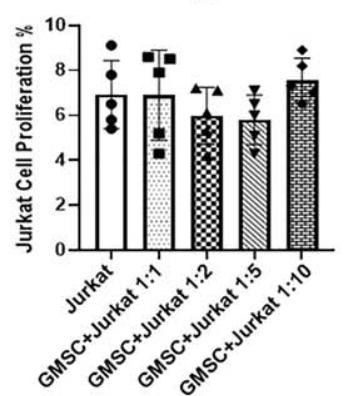

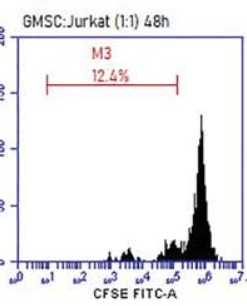

Proliferation\% $12 \mathrm{~h}$
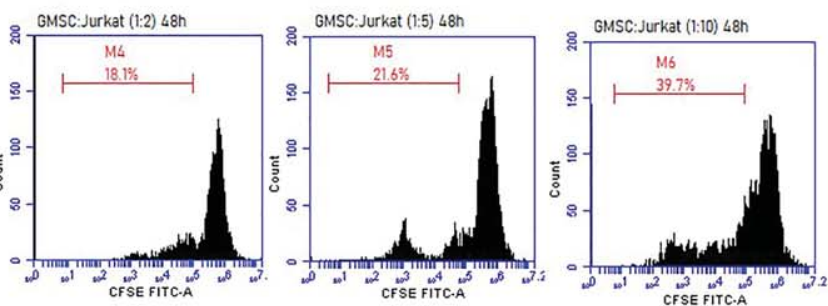

Proliferation\%

$24 \mathrm{~h}$

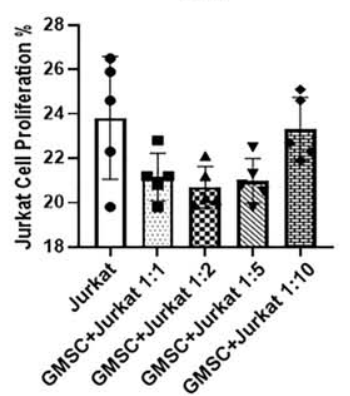

Proliferation\% $48 \mathrm{~h}$

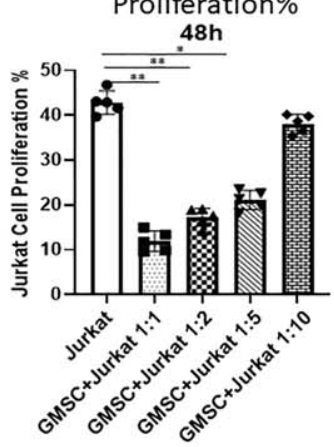

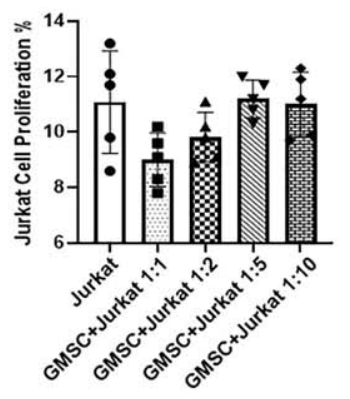

Figure 2. Lymphoproliferative response of the Jurkat cells. CFSE-labeled Jurkat cells were stimulated with anti-CD3/anti-CD28 for specific T lymphocyte responses. The Jurkat cells cultured alone showed high proliferation ratio within 24 and 48 hours of culture periods. GMSCs significantly reduced the lymphoproliferative response of the Jurkat cells in I:I, I:2, and I:5 (GMSC:Jurkat) ratios. The minimum suppressive response for the proliferation of Jurkat cells cultured with GMSCs was found to be in 1:5 ratios. Statistical analysis of the proliferation of the Jurkat cells in the presence and absence of GMSCs. The proliferative response of the Jurkat cells with GMSCs did not significantly change in 6, 12, and 24 hours culture periods but tended to decrease in 24 hours. GMSCs significantly decreased proliferation ratio of the Jurkat cells in 48 hours of culture period in $1: 1,1: 2$, and 1.5 ratios $\left(P<.01,<.01\right.$, and $<.05$, respectively). ${ }^{*} P<.05$, ${ }^{* *} P<.01$, ${ }^{* * *} P<.005$, and ${ }^{* * * *} P<.001$.

compared to 48 hours of culture period of the Jurkat cells alone $(\mathrm{II} .8 \pm 2.4,16.2 \pm 4.3$, and $21.4 \pm 3.7$, respectively $)(P<.0 \mathrm{l},<.0 \mathrm{l}$, and $<.05)$.

The Jurkat cells cultured with GMSCs in the ratio of $1: 10$ tended to decrease the proliferation ratio $(39.6 \pm 4.8)$ in 48 hours of culture period, but no significant difference was observed when compared with 48 hours of the Jurkat cell cultures alone $(P>.05)$ (Figure 2).

The minimum suppressive ratio of the cocultured cells was found to be 1:5 (GMSCs:Jurkat). Therefore, we continued the cell cultures in 1:5 ratio with the culture periods of 6, 12,24, and 48 hours for the analysis of apoptosis, intracellular IL-2 cytokine secretion, CD4+CD25+FoxP3+ T cell frequency, and TNFRI and TNFR2 expressions.

\section{GMSCs Increased Early Apoptosis and Necrosis in the Jurkat Cells}

The analysis for apoptosis was performed by staining the cells with Annexin $V$ and 7AAD antibodies to analyze early and late apoptosis and necrosis in the Jurkat cells. The Jurkat cells cultured alone showed low ratio of early apoptosis (6 hours: I.I \pm 0.2 , 12 hours: $10.4 \pm 0.5$, 24 hours: $11.8 \pm 0.3$, and 48 hours: $11.9 \pm$ 0.2 ), late apoptosis (6 hours: $3.2 \pm 0.3,12$ hours: $0.6 \pm 0.2$, 24 hours: $2.0 \pm 0.4$, and 48 hours: $1.7 \pm 0.4$ ), and necrosis (6 hours: $1.6 \pm 0.1,12$ hours: $4.8 \pm 0.4,24$ hours: $3.3 \pm 0.5$, and 48 hours: $6.6 \pm 0.3)$. GMSCs significantly increased the early apoptosis and necrosis of the Jurkat cells (early apoptosis: 6 hours: $7.5 \pm 0.4, P<.05,12$ hours: $16.8 \pm 0.3, P<.05,24$ hours: $55.6 \pm 5.7, P<.001$, and 48 hours: $59.2 \pm 4.8, P<.001$; necrosis: 6 hours: $8.7 \pm 0.6, P<.01$, 12 hours: $13.7 \pm 1.9, P<.01,24$ hours: 7.8 $\pm 3.2, P<.005$, and 48 hours: $15.6 \pm 4.2, P<.01)$ compared to those in the Jurkat cells alone (Figure 3 ).

\section{Intracellular IL-2 Secretion in Jurkat Cells Reduced with GMSCs} $\mathrm{IL}-2$ is a cytokine that induces activation and proliferation of $\mathrm{T}$ cells. In this study, we analyzed intracellular IL-2 secreting $T$ cells to determine the effect of GMSCs on the activation of the Jurkat cells. The Jurkat cells were stained with anti-CD3 for cell surface marker of lymphocytes, and intracellular staining was done with anti-IL-2 antibody. The Jurkat cells cultured alone showed a high ratio of intracellular IL-2 in 24 and 48 hours of culture periods $(14.1 \pm 1.7,16.3 \pm 0.8$, respectively). GMSCs significantly decreased intracellular IL-2 secreting cells in 24 and 48 hours of cocultures, compared to cultures of the Jurkat cells alone (24 hours: $9.2 \pm 0.4, P<.01$, 48 hours: $8.3 \pm 0.2, P<.005$, respectively) (Figure 4 ).

\section{Frequency of FoxP3 Expressing CD4+CD25+ Tregulatory Cells} in the Jurkat Cell Population Tended to Increase with GMSCs

We evaluated the effect of GMSCs on FoxP3 expressing CD4+CD25+ T regulatory cell population in Jurkat cells. The cultured cells first analyzed for cell surface expression of CD4 and CD25, and intracellular FoxP3 staining was performed to determine $\mathrm{CD} 4+\mathrm{CD} 25+\mathrm{FoxP} 3+\mathrm{T}$ regulatory cell frequency. The cultured Jurkat cells showed low frequency of CD4+CD25+FoxP3+ $T$ cell population when cultured alone in $6,12,24$, and 48 house of culture periods ( 6 hours: $1.3 \pm 0.2$, 12 hours: $2.2 \pm 0.1$, 24 hours: $2.6 \pm 0.6$, and 48 hours: $3.3 \pm 1.1$ ). GMSCs tended to increase the CD4+CD25+FoxP3+ cell population in 24 and 48 hours cultures, compared to those in Jurkat cells alone cultures ( 6 hours: $1.6 \pm 0.3,12$ hours: $3.1 \pm 0.2$, 24 hours: 

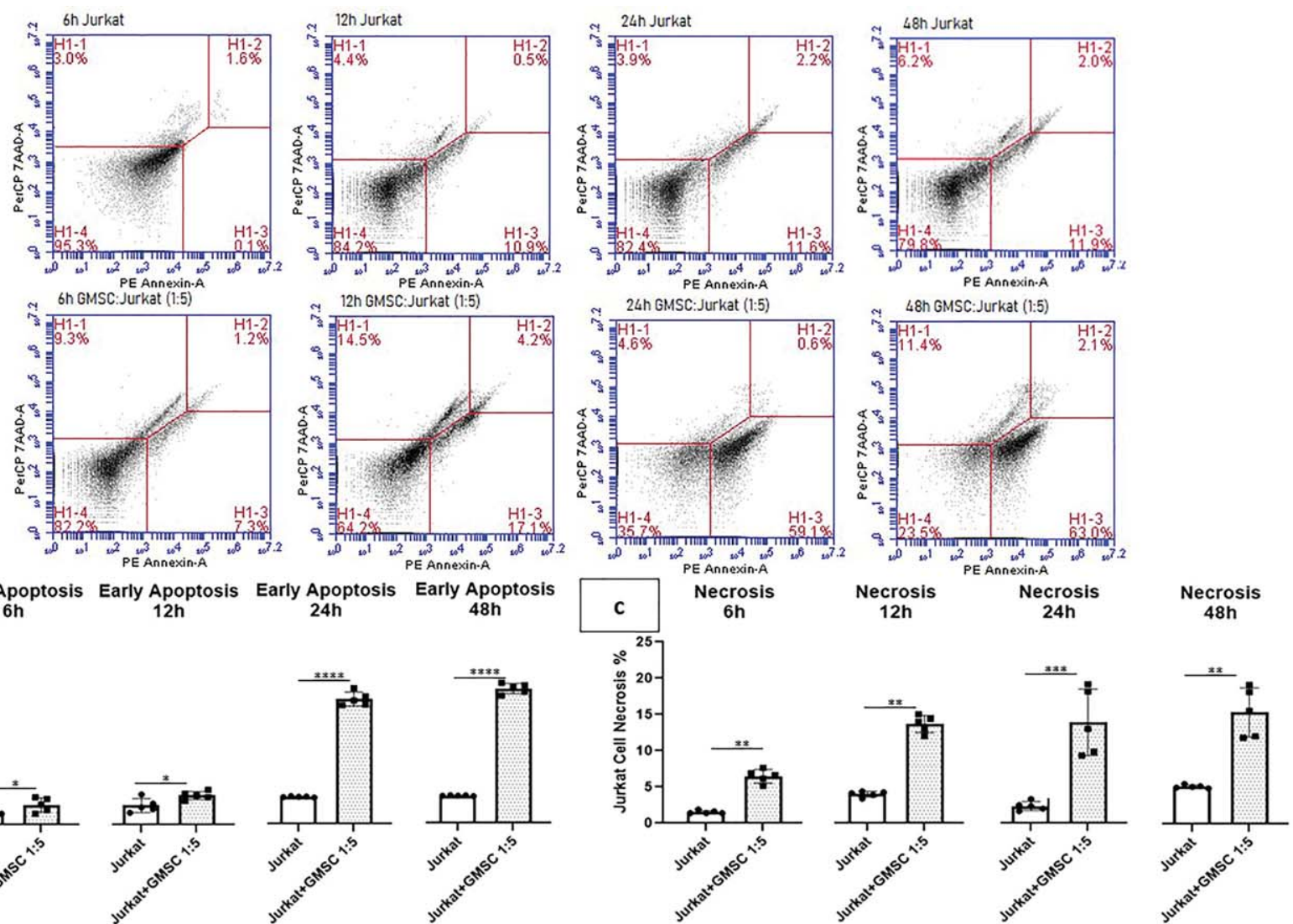

Figure 3. Analysis for apoptosis, necrosis, and cell survival of the Jurkat cells in the presence and absence of GMSCs. Representative analysis for Annexin $V$ and 7AAD in the Jurkat cells. Analysis of quadrants was done as following: lower left: cell survival; lower right: early apoptosis; upper right: Iate apoptosis; upper left: necrosis. Statistical analysis for apoptosis and necrosis. The Jurkat cells showed low ratio of early and late apoptosis and necrosis when cultured alone in 6, 12,24, and 48 hours of the culture periods. GMSCs significantly increased early apoptosis and necrosis in Jurkat cells compared to the Jurkat cell cultures alone (early apoptosis: 6 hours: $7.5 \pm 0.4, P<.05,12$ hours: $16.8 \pm 0.3, P<.05,24$ hours: $55.6 \pm 5.7, P<.001$, and 48 hours: $59.2 \pm 4.8, P<.001$; necrosis: 6 hours: $8.7 \pm 0.6, P<.01$, 12 hours: $13.7 \pm 1.9, P<.01,24$ hours: $7.8 \pm 3.2, P<.005$, and 48 hours: $15.6 \pm 4.2, P<.01) .{ }^{*} P<.05,{ }^{* *} P<.01,{ }^{* * *} P<.005$, and ${ }^{* * * *} P<.001$.

$3.2 \pm 0.1$, and 48 hours: $3.6 \pm 0.2)$, but no significant difference was observed $(P>.05)$ (Figure 5).

\section{GMSCs Increased TNFR2 Expression on the Jurkat Cells}

We analyzed TNFRI and TNFR2 expressions for the apoptotic signals on the Jurkat cells. The Jurkat cells showed low expression of TNFRI and TNFR2 when cultured alone ( 6 hours: TNFRI: $1.0 \pm 0.2$, TNFR2: $1.9 \pm 0.5$, 12 hours: TNFRI: $1.5 \pm 0.3$, TNFR2: $2.1 \pm$ $0.4,24$ hours: TNFRI: $2.9 \pm 0.8$, TNFR2: $2.6 \pm 0.1$, and 48 hours: TNFRI: $2.1 \pm 0.4$, TNFR2: $3.0 \pm 0.5$ ). TNFR2 expression was not significantly changed in the Jurkat cells in 6 hours culture period with GMSCs, but GMSCs significantly increased TNFR2 expression on the Jurkat cells in 12,24, and 48 hours of culture periods ( 6 hours: $3.5 \pm 0.7, P>.05,12$ hours: $19.7 \pm 3.8, P<.001$, 24 hours: $26.9 \pm 4.2, P<.001$, and 48 hours: $28.9 \pm 3.1, P<.001$, respectively). There was no significant difference in TNFRI expression in cultures of Jurkat cells with GMSCs $(P>.05)$ (Figure 6).

\section{DISCUSSION}

T-cell acute lymphoblastic leukemia is one of the most common $\mathrm{T}$ lymphocyte-related malignancies, accounting for $10-25 \%$ of all cancer cases in children and $25 \%$ in adults. ${ }^{8}$ Although many treatments have been developed on tumor cells, the effectiveness of the treatments is still not sufficient, and innovative therapeutic approaches are needed. In this study, we investigated the antiproliferative and apoptotic effects of GMSCs on the Jurkat cells as T-cell acute lymphoblastic leukemia cell line. The results showed that GMSCs downregulated proliferative response of the Jurkat cells by enhancing early apoptosis and downregulating IL-2 secretion. In addition, GMSCs upregulated TNFR2 expression on the Jurkat cells; this may be evidence of an apoptotic effect of GMSCs on Jurkat cells through a member of the TNFR family.

GMSCs are the most easily isolated stromal adult stem cells with a high doubling time and high anti-inflammatory effect compared to many other sources. In a previous study, it was demonstrated that GMSCs inhibit the proliferation of oral cancer cells by inducing proapoptotic signals with the soluble factors. ${ }^{3}$ In addition, bone marrow or Wharton's jelly MSCs have an anti-tumoral effect on lung cancer cells, colorectal cancer, or bladder tumor cells by inducing macrophages in the regulatory phenotype or inducing apoptosis of cancer cells. We, therefore, in the present study, investigated the effects of GMSCs on the proliferative responses, apoptosis, and T regulatory cell frequency in the Jurkat cells. 

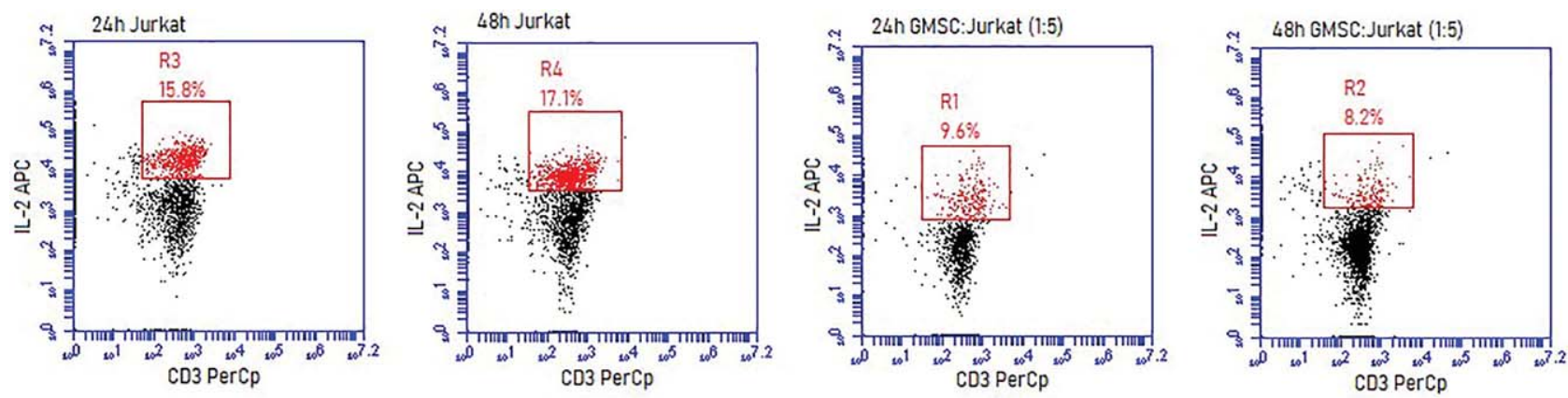

\section{IL-2 Secretion $6 \mathrm{~h}$}

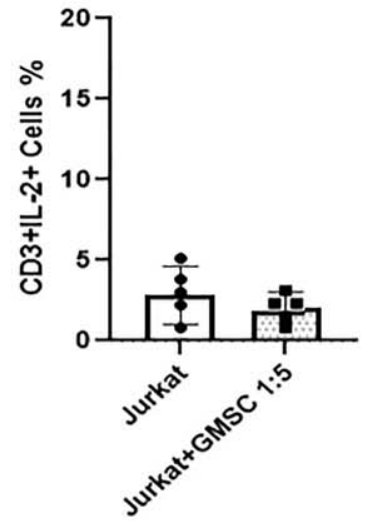

\section{IL-2 Secretion} $12 \mathrm{~h}$

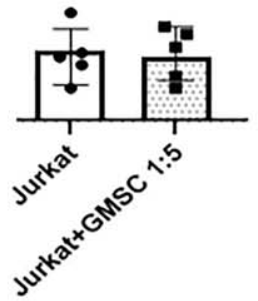

IL-2 Secretion 24h

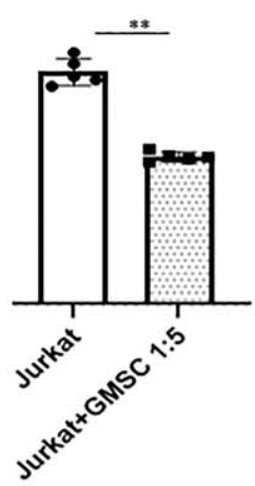

IL-2 Secretion $48 \mathrm{~h}$

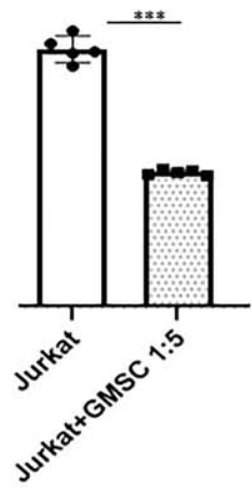

Figure 4. Analysis of IL-2 secreting CD3+ cells. The representative analysis for IL-2 secreting Jurkat cells by flow cytometry. The Jurkat cells were gated as CD3 + cell population, and IL-2 secretion of CD3+ cells was analyzed by gating double positive area in the dot plot analysis. Statistical analysis for IL-2 secreting CD3+ cells. IL-2 secretion significantly decreased in Jurkat cells when cultured with GMSCs in 24 and 48 hours of culture periods, compared to those in the Jurkat cell cultures alone ( 24 hours: $P<.01$ and 48 hours: $P<.005$, respectively). ${ }^{*} P<.05,{ }^{* *} P<.01,{ }^{* * *} P$ $<.005$, and ${ }^{* * * *} P<.001$

Avoiding apoptosis is an important feature of cancer and is vital for maintaining a balance between proper apoptotic signaling, cell survival, and cell death. The dysregulation of apoptotic pathways in cancer cells not only promotes tumor formation but can also make cancer cells resistant to anticancer agents. ${ }^{7,10}$ However, antitumor effects of MSCs are still controversial. Additionally, different results obtained in studies may vary depending on the source of MSCs or the type and stage of cancer. In this study, we demonstrated that increasing concentrations of GMSCs and increased expression of TNFR2 in Jurkat cells, which may be directly involved in cell death. Targeting TNFR2 is crucial in cancer therapy since it controls both immunosuppression and angiogenesis in cancer cells. 11,12 The results showed that the proliferation of the Jurkat cells decreased with GMSCs in concentration-dependent manner. The inhibition of proliferative responses found to be the increasing rate of early apoptosis and necrosis of the Jurkat cells.

Regulatory $T$ cells are known to suppress immune functions through various mechanisms such as the production of IL-I0 as an immunosuppressive cytokine. T regulatory cells also provide immunoregulation by consuming the cytokine IL-2 produced by activated T lymphocytes in the inflammatory environment. ${ }^{13}$ In the present study, we evaluated the effect of GMSCs on the frequency of CD4+CD25+FoxP3+ T regulatory cells within the Jurkat cells. Although no significant difference was observed, the results showed that GMSCs tended to increase the generation of FoxP3 expressing CD4+CD25+ regulatory T cell population in the Jurkat cell line. One reason for the inhibitory effect of GMSCs on Jurkat cells may be the slight increase in T regulator cells.

IL-2 is a pleiotropic cytokine that promotes the differentiation of both pro- and anti-inflammatory $\mathrm{T}$ cells. Additionally, IL-2 supports the proliferative expansion of $T$ lymphocytes and the proliferation of antigen-specific $T$ cell clones. ${ }^{4} \mathrm{IL}-2$ transduced MSCs can secrete or produce various antitumor agents or mediators, which could prevent and keep from metastases. ${ }^{15}$ Studies to date indicate that MSCs can downregulate IL-2 production from $T$ lymphocytes through induction of CD25 cleavage and have an essential role in the inhibition of $T$ cell proliferative responses. ${ }^{16-18}$ Previous studies indicate that IL-2 produced during inflammatory responses in the microenvironment may further enhance the immunosuppressive response effect of MSCs. We investigated the effect of GMSCs on IL-2 production in Jurkat cells and found that GMSCs strongly inhibit IL-2 production in Jurkat cells. The proliferative response of Jurkat cells cocultured with GMSCs may have been inhibited by down-regulation of IL-2 production. 

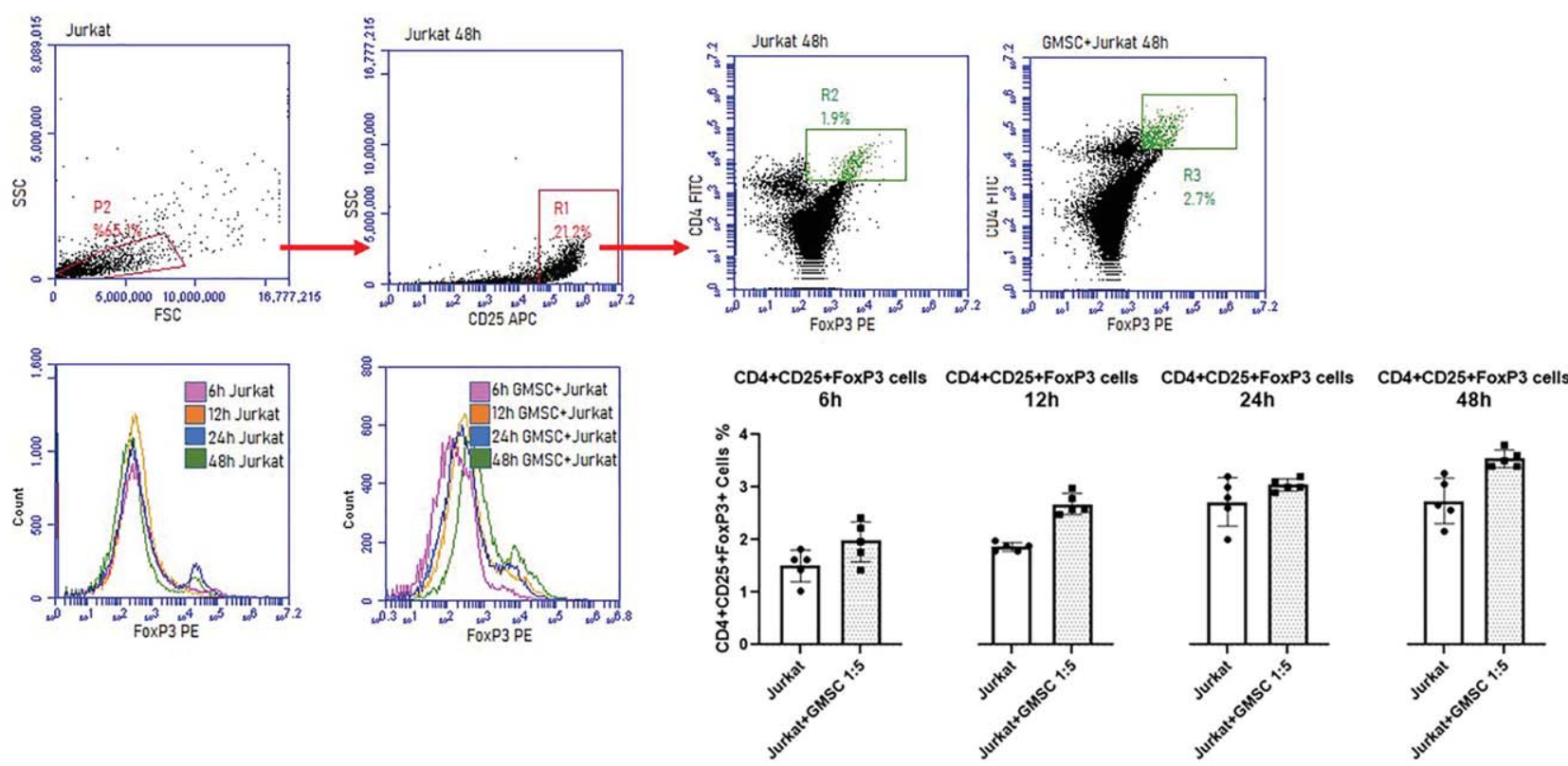

Figure 5. FoxP3 expressing CD4+CD25+ T regulatory cells. The Jurkat cells were analyzed for the effect of GMSCs on the generation of $T$ regulatory cells in the Jurkat cell cultures. Gating strategy for flow cytometry analysis of the CD4+CD25+FoxP3+ cell population in the Jurkat cells. Analysis was done by gating CD25+ cells in the total lymphocyte population. CD25+ cells were analyzed for CD25+FoxP3+ cells. Comparative histogram analysis for the Jukat cells in the presence and absence of GMSCs. Statistical analysis of the CD4+CD25+FoxP3+ cells in the Jurkat cells in the presence and absence of GMSCs. GMSCs tended to increase T regulatory cell population in the Jurkat cells, but no significant difference was observed when compared with Jurkat cell cultures alone $(P>.05)$.
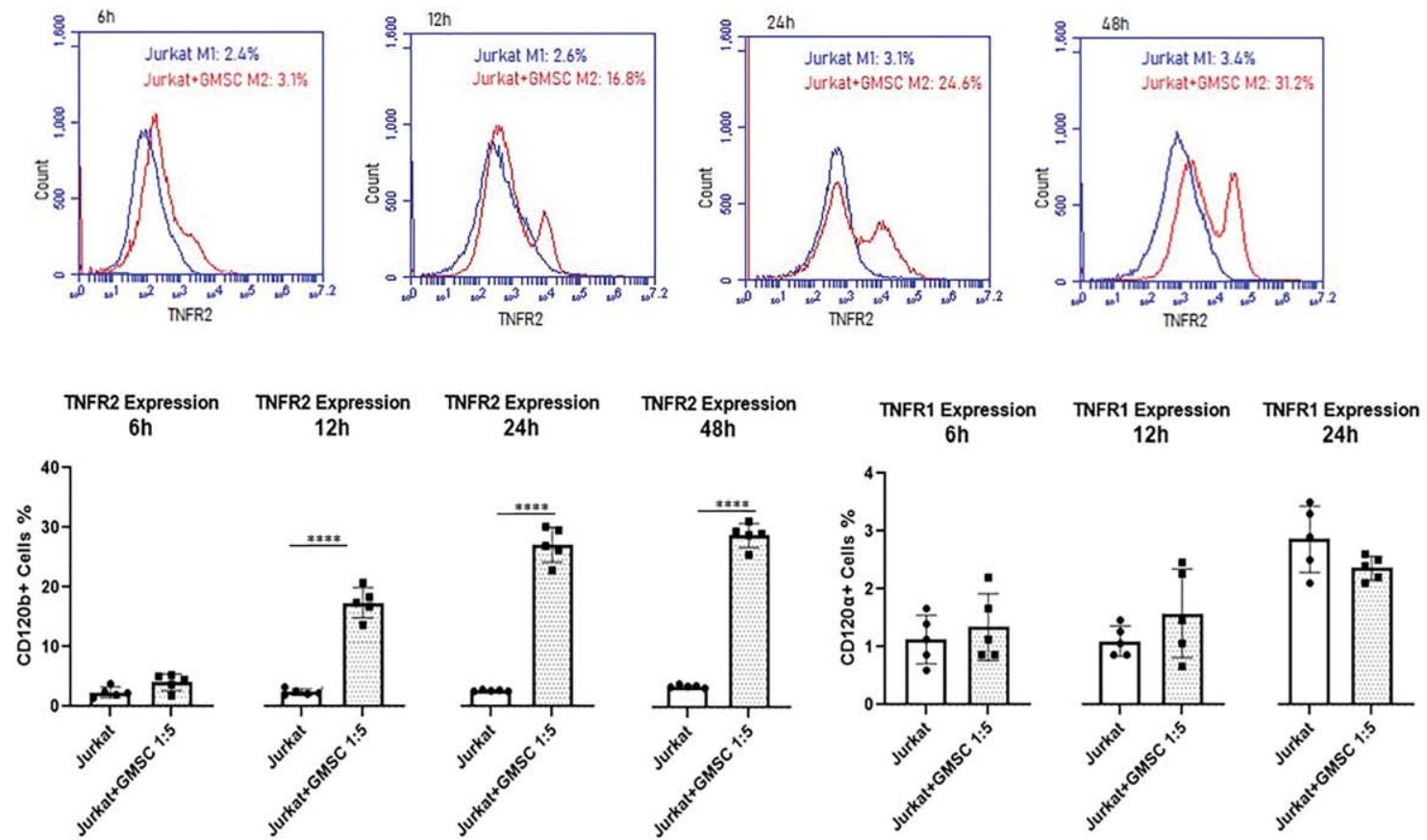

TNFR1 Expression
$48 \mathrm{~h}$

Figure 6. TNFRI and TNFR2 expressions on the Jurkat cells. One of the apoptotic pathways was analyzed for the Jurkat cells cultured with or without GMSCs. The comparative histogram analysis for TNFR2 expression on the Jurkat cells. Statistical analysis for TNFRI or TNFR2 expressions of Jurkat cells in the presence and absence of GMSCs. GMSCs significantly increased TNFR2 expression on the Jurkat cells in 12, 24, and 48 hours of culture periods ( 12 hours: $19.7 \pm 3.8, P<.001$, 24 hours: $26.9 \pm 4.2, P<.001$, and 48 hours: $28.9 \pm 3.1, P<.001)$. ${ }^{* * * *} P<.001$.

In conclusion, results showed that GMSCs downregulate the proliferative response of the Jurkat cells by increasing apoptosis and necrosis and reducing $\mathrm{IL}-2$ production by the Jurkat cells. In addition, GMSCs upregulate the expression of TNFR2 on the Jurkat cells, which, in turn, may promote apoptosis of these cells. Further in vitro or in vivo studies can be conducted with GMSCs for the cell-based treatment of T-cell acute lymphoblastic leukemia. 
Ethics Committee Approval: Ethical committee approval was received from the Muğla Sıtkı Koçman University Clinical Research Ethics Committee (I0/VII 0I.10.2020).

Informed Consent: Informed consent was obtained from all participants.

Peer-review: Externally peer-reviewed.

Author Contributions: Design - D.G.; Data Collection and/or Processing D.G., M.S.K., S.S.; Analysis and/or Interpretation - D.G., M.S.K.

Acknowledgment: The present study was funded by the Muğla Sıtkı Koçman University Research Projects (Project No: 20/106/02/3/4).

Conflict of Interest: The authors have no conflicts of interest to declare.

Financial Disclosure: The present study was funded by Muğla Sıtkı Koçman University Research Projects (Project No: 20/106/02/3/4).

\section{REFERENCES}

I. Yang T, Yao S, Zhang X, Guo Y. Andrographolide inhibits growth of human T-cell acute lymphoblastic leukemia Jurkat cells by downregulation of PI3K/AKT and upregulation of p38 MAPK pathways. Drug Des Dev Ther. 2016;10:1389-1397. [CrossRef]

2. Zhu $X L$, Jiang $L, Q \cup F$, Wang $Z Y$, Zhao LM. Inhibitory effect of Embelin on human acute T cell lymphoma Jurkat cells through activation of the apoptotic pathway. Oncol Lett. 2015;10(2):921-926. [CrossRef]

3. Ji $X$, Zhang $Z$, Han $Y$, et al. Mesenchymal stem cells derived from normal gingival tissue inhibit the proliferation of oral cancer cells in vitro and in vivo. Int J Oncol. 2016;49(5):2011-2022. [CrossRef]

4. Fawzy El-Sayed KM, Dörfer CE. Gingival mesenchymal stem/progenitor cells: A unique tissue engineering gem. Stem Cells Int. 2016;20l6:I-16. [CrossRef]

5. Lin W, Huang L, Li Y, et al. Mesenchymal stem cells and cancer: Clinical challenges and opportunities. Biomed Res Int. 2019;2019:1-12. [CrossRef]

6. Chang Al, Schwertschkow AH, Nolta JA, Wu J. Involvement of mesenchymal stem cells in cancer progression and metastases. Curr Cancer Drug Targets. 2015;15(2):88-98. [CrossRef]

7. Lee MW, Ryu S, Kim DS, et al. Mesenchymal stem cells in suppression or progression of hematologic malignancy:
Current status and challenges. Leukemia. 2019;33(3):597-611. [CrossRef]

8. Baghbani E, Khaze $V$, Sadreddini $S$, et al. PTPN22 silencing in human acute T-cell leukemia cell line (Jurkat cell) and its effect on the expression of miR-18la and miR-18lb. Adv Pharm Bull. 2018;8(2):277-282. [CrossRef]

9. Wang $Q, L i T, W u W$, Ding $G$. Interplay between mesenchymal stem cell and tumor and potential application. Human Cell. 2020;33:444458. [CrossRef]

10. Plati J, Bucur O, Khosravi-Far R. Apoptotic cell signaling in cancer progression and therapy. Integr Biol (Camb). 2011;3(4):279-296. [CrossRef]

II. Naserian S, Abdelgawad ME, Afshar Bakshloo M, et al. The TNF/ TNFR2 signaling pathway is a key regulatory factor in endothelial progenitor cell immunosuppressive effect. Cell Commun Signal. 2020;18(I):94. [CrossRef]

12. Depuydt B, van Loo G, Vandenabeele P, Declercq W. Induction of apoptosis by TNF receptor 2 in a T-cell hybridoma is FADD dependent and blocked by caspase- 8 inhibitors. J Cell Sci. 2005; II8(P+ 3):497-504. [CrossRef]

13. Ohue Y, Nishikawa H. Regulatory T (Treg) cells in cancer: Can Treg cells be a new therapeutic target? Cancer Sci. 2019;110(7):20802089. [CrossRef]

14. Ross SH, Cantrell DA. Signaling and function of interleukin-2 in T lymphocytes. Annu Rev Immunol. 2018;36:41I-433. [CrossRef]

15. Chulpanova DS, Solovyeva VV, James V, Arkhipova SS, et al. Human mesenchymal stem cells overexpressing interleukin 2 can suppress proliferation of neuroblastoma cells in co-culture and activate mononuclear cells in vitro. Bioengineering (Basel). 2020;7(2):59. [CrossRef]

16. Park MJ, Shin JS, Kim YH, Hong SH, et al. Murine mesenchymal stem cells suppress $T$ lymphocyte activation through IL-2 receptor $\alpha$ (CD25) cleavage by producing matrix metalloproteinases. Stem Cell Rev Rep. 20ll;7(2):38I-393. [CrossRef]

17. Yoo HS, Lee K, Na K, et al. Mesenchymal stromal cells inhibit CD25 expression via the mTOR pathway to potentiate T-cell suppression. Cell Death Dis. 2017;8(2):e2632-e2632. [CrossRef]

18. Davies LC, Heldring N, Kadri N, Le Blanc K. Mesenchymal stromal cell secretion of programmed death-I ligands regulates $T$ cell mediated immunosuppression. Stem Cells. 2017;35(3):766-776. [CrossRef] 